\title{
22 Zusammenfassende Vertiefung am Beispiel mythischer Figuren
}

\subsection{Polymorphe und polystrate Protagonisten}

Selbst jetzo, welche denn ich sei, ich weiß es nicht.

Helena in Goethes Faust II, 3. Akt, Vers 8875

Im Verlauf der Arbeit ist deutlich geworden, wie wichtig es ist, bei der Untersuchung von medialen Konkretionen mythischer Stoffvarianten nicht nur deren horizontale Hylemstruktur zu analysieren, und zwar ohne nach Art bestimmter narratologischer, formalistisch-strukturanalytischer oder strukturalistischer Ansätze einzelne stoffliche Züge vorwegnehmend als funktional oder semantisch bedeutsam $\mathrm{zu}$ interpretieren (Kapitel 5.1 und 5.2), sondern durch die Unterscheidung von Schichten zusätzlich die vertikale Tiefendimension solcher Stoffvarianten in den Blick zu nehmen.

Bei einer stoffwissenschaftlichen Herangehensweise kommt es auf die Umsetzung der Erkenntnis an, daß es sich um die Analyse nicht medialer Konkretionsformen, also z. B. textlicher oder bildlicher Strukturen handelt, sondern um die Analyse von Inhalten, die es erst aus den jeweiligen medialen Konkretionen zu extrahieren, in die Form von Hylemen zu überführen und in ihre natürliche chronologische Abfolge zu bringen gilt. Diese Stoff-Rekonstruktion ist ein komplexer Prozeß (Kapitel 5.3 und 6.3).

Im Hinblick auf eine Analyse von Stoffschichten hat sich gezeigt, daß Stoffe in ständigem Kontakt zu anderen Stoffen stehen (Kapitel 13.2); daß hinter diesen Kontakten polemische Auseinandersetzungen stehen (Kapitel 18.4.1); daß die vielfältigen und kämpferischen Kontakte zwischen mythischen Stoffen bestimmten Gesetzmäßigkeiten folgen und bestimmten Arten zugeordnet werden können (Kapitel 13.3 und 14); daß solche Stoff-Stoff-Kontakte in Konkretionen mythischer Stoffvarianten Spuren hinterlassen, aufgrund derer man Rückschlüsse auf den Vorgang solcher Stoffkontakte ziehen kann; und daß es neben der Beobachtung bestimmter Muster von Stoff-Stoff-Interferenzen weitere belastbare Kriterien für die Identifizierung solcher Spuren gibt, nämlich Inkonsistenzen auf formaler und logischer Ebene (Kapitel 16) und Wertungen bzw. Hierarchisierungen als typische gedankliche Muster auf semantischer Ebene (Kapitel 19), die sich wiederum in bestimmten Erzähltaktiken konkret manifestieren (Kapitel 20). Mit Hilfe der 
durch distinkte Kriterien bestimmbaren Indizien lassen sich in mythischen Stoffvarianten verschiedene Strata ausmachen. Daß in medialen Konkretionen mythischer Stoffvarianten grundsätzlich mit verschiedenen Schichten zu rechnen ist, ist aufgrund des Phänomens der Interhylität mehr als wahrscheinlich.

Wenn man die Komplexität eines stofflichen Gebildes wie die eines Mythos, die durch die Vielzahl der möglichen Varianten sowie durch Stoffkontakte und mit den Kontakten verbundene Deutungsmachtkonflikte bedingt ist, nicht allzu sehr simplifizieren will, dann ist ein solcher Stoff nur als ein polymorphes und je nach einzelner Variante polystrates Gebilde begreifbar. Mythen sind variantenreiche Stoffpotentiale und je nach Varianten mehrfach geschichtete Produkte, deren inhaltliche Strukturen erst durch eine Hylemanalyse rekonstruiert werden müssen und deren Strata bzw. Tiefendimensionen dann durch eine Stratifikationsanalyse aufgeschlüsselt werden können.

All diese Beobachtungen besitzen nun aber nicht nur für einzelne Stoffvarianten insgesamt Gültigkeit, also für zusammenhängende Hylemsequenzen, sondern auch für eine bestimmte und wichtige Gruppe unter den Hylemelementen, und zwar für die in mythischen Stoffen handelnden Figuren. Fast alle Eigenarten und Merkmale von mythischen Stoffen und ihren Varianten lassen sich auch auf die in diesen Stoffen auftretenden Protagonisten übertragen, und im Folgenden sollen die bisher bei der Analyse mythischer Stoffe bzw. Stoffvarianten gewonnenen Ergebnisse nicht nur resümierend genannt sein, sondern zugleich auf das Untersuchungsfeld mythischer Figuren angewendet werden.

Wie ein Erzählstoff, so sind auch die in diesen Stoffen auftretenden Figuren nicht mit den unterschiedlichen medialen Konkretionen gleichzusetzen, in denen sie sich darbieten, sondern es handelt sich dabei um Vorstellungsinhalte, die sprachlich, bildlich oder auf noch andere Weisen unterschiedlich konkretisiert werden können und müssen. Wie ein mythischer Erzählstoff im Großen, so ist auch eine mythische Figur im Kleinen polymorph und damit ein unscharfes Gebilde; dies wurde in Kapitel 12.2 bereits an der Figur des Herakles des Näheren ausgeführt. Genauso wenig wie es „den“ Stoff von Niobes Hybris gegenüber Leto gibt, genauso wenig existiert dementsprechend „die“ Niobe oder „der“ Herakles, und man kann hier noch einmal in aller Kürze die verschiedenen Versuche Revue passieren lassen und zugleich verwerfen, die darauf aus sind, einen mythischen Stoff und dementsprechend auch eine Figur in mythischen Stoffen nicht als eine „Vielform“, sondern als eine „Einform“ zu begreifen (Kapitel 4.1-4). Es hilft wenig, sich an einer Glanzversion der Niobe zu orientieren, wie sie bspw. in Ovids Metamorphosen präsentiert wird; man wird hinsichtlich der Gestalt der Niobe weder eine Minimalversion, einen absolut festen und unverrückbaren „Figuren- 
kern“ eindeutig bestimmen können, der gewissermaßen als das Eigentliche gelten kann, während anderes „nur“ oder „spätere“ Zutat ist, noch wird man hinreichend belastbare Kriterien entwickeln können, die es erlauben, eine bestimmte Darstellung oder ein festes Set an Eigenschaften als klar definierbare Standardversion der Niobe hinzustellen, und auch zu einer Urversion der Niobe wird man nicht mehr vorstoßen können, schon gar nicht, wenn man ähnlich wie bei der Unterscheidung zwischen konkretem Stoff und Stoffschema so auch bei der Gestalt der Niobe berücksichtigt, daß hinter verschiedenen konkreten Zügen dieser Gestalt bestimmte Typica stecken, die auch in anderen, vergleichbaren Figuren realisiert gewesen sein und die Konzeption der Niobe-Gestalt beeinflusst haben können ${ }^{1}$.

Und schließlich führt auch der Ansatz nicht zum Ziel, die Maximalversion, die Gesamtheit aller zur Verfügung stehenden Niobe-Konkretionen als hinreichende Lösung für die Bestimmung „der“ Niobe anzusehen (vgl. Kapitel 4.5). Denn wie mythische Stoffe, so stellen auch mythische Protagonisten nicht etwas fest Umreißbares dar, sondern in jeder Figur steckt ein im Prinzip unauslotbares Potential (s. Kapitel 4.6), dem durch das Wechselspiel zwischen zentrifugalen und zentripetalen Kräften im Traditionsprozeß zwar eine gewisse Formkonstanz eignet; aber diese Form besitzt keine starre Kontur oder gar festgelegte Schärfe (vgl. Kapitel 12.1-2). Ähnlich wie bei Stoffen, so können auch bei mythischen Figuren viele nähere Bestimmungen der Gestalt verändert oder ausgetauscht werden, nur nicht alle auf einmal, und wie bei Stoffen so ist auch bei Figuren die Schwelle individuell verschieden, ab der man die Darstellung einer Figur als gerade noch erkennbare Verfremdung eben dieser Figur zu begreifen in der Lage oder bereits nicht mehr in der Lage ist (Kapitel 12.3).

Eine mythische Figur existiert aber nun nicht nur in Form vieler verschiedener Varianten (wie ein mythischer Stoff), sie ist nicht nur polymorph, sondern sie ist (wie eine einzelne mythische Stoffvariante) in der Regel auch polystrat, das heißt in jeder medial konkretisierten Einzelvariante ein zusammengesetztes, vielschichtiges, von vielen verschiedenen Einflußfaktoren bestimmtes Gebilde. Denn keine Konkretion einer mythischen Figur entsteht in einem luftleeren Raum, und sie wird auch nicht von einem einzelnen Maler auf Leinwand oder von einem einzelnen Autor am Schreibtisch neu geschaffen, sondern sie führt ein von einzelnen Schaffenden unabhängiges Vorleben ${ }^{2}$ und noch dazu ein weit über einen einzelnen Stoff hinausgehendes Eigenleben. Die Überlegungen zum „Tod des

1 Zur Unterscheidung von Stoff und Stoffschema s. Kapitel 7.3.

2 S. zu dem vergleichbaren, allerdings rein textbezogenen Konzept der „Interfiguralität“ von Müller (1991) die Ausführungen in Kapitel 13.2. 
Autors “ und das Phänomen der Interhylität spielen daher nicht nur für mythische Stoffe, sondern auch für mythische Figuren eine wichtige Rolle (s. dazu Kapitel 13.1-2). Ihr unscharfes „Profil“ konstituiert sich aus verschiedensten Traditionen und bedeutungstragenden Elementen der eigenen Kultur und von Kulturen, mit denen diese direkt oder indirekt in Kontakt steht ${ }^{3}$.

Ähnlich wie bei Stoffen, so zielt auch bei Figuren der Interhylitätsbegriff aber nicht nur auf ein allgemeines Vernetzt-Sein in einem unspezifischen Sinn. Wie bei Stoffen die Untersuchung des Kontakts speziell mit anderen Stoffen zu fruchtbaren Ergebnissen geführt hat, lohnt es sich bei Figuren, besonders auf Berührungspunkte mit und Verhältnisse $\mathrm{zu}$ anderen Figuren $\mathrm{zu}$ achten, und auch dies wiederum nicht in einem unspezifischen Sinn, sondern im Hinblick auf hauptsächlich polemische Abgrenzungen und Auseinandersetzungen zwischen verschiedenen Figurenkonzeptionen (s. dazu die Kapitel 18.4.1, 18.4.3 und 19.1). Ebenso wie bei Stoffen kann es auch auf der Ebene der Figuren zu Figuren-Interferenzen aufgrund von konzeptuellen Parallelen, von Ähnlichkeiten bzw. Gleichheiten von Namen oder aufgrund der Sogwirkung oder Ausstrahlung von besonders prominenten Gestalten kommen (Kapitel 13.3 und 14), und all diese Interferenzen spielen sich nicht in einem Machtvakuum ab, sondern in einer Arena, in der Deutungskonflikte kämpferisch ausgetragen werden.

Die Folgerungen, die sich aus diesen verschiedenen Beobachtungen für eine Einordnung und Interpretation mythischer Figuren ergeben, sind demgemäß ganz ähnlich wie die, die für die Deutung mythischer Stoffe herausgearbeitet wurden. Das Streben nach Deutungshoheiten impliziert in der Regel Machtansprüche, Machtansprüche können zu Konflikten führen, und diese Konflikte hin-

3 Die „Mehrdimensionalität“ mythischer (und z.T. auch sagenhafter) Figuren beruht zwar auch, aber nicht primär auf dem Kunstschaffen eines einzelnen Autors. Wenn Suerbaum, 1999, 205, mit Bezug auf Vergils Aeneis schreibt: „Vergil hat seine Figuren nicht einfach aus irgendwelchen mythisch-historischen Quellen oder von bestimmten literarischen Vorlagen übernommen“, so ist dazu anzumerken, daß selbst wenn Vergil dies hätte tun wollen, dies alles andere als „einfach“ gewesen wäre, denn die Figuren lagen in diesen Quellen oder Vorlagen nicht in einer eindeutigen und damit „gebrauchsfertigen“ Gestalt vor. Nach Suerbaum (ebd.) besteht Vergils Kunst „darin, Anregungen von verschiedenen Seiten, nicht zuletzt von verschiedenen Partien und Personen in den beiden Werken Homers, aufzunehmen und zu verschmelzen." Dies ist aber nicht nur Kunst, sondern bis zu einem gewissen Grad unumgänglich, nicht nur aufgrund der Polymorphie jeder einzelnen Figur, sondern auch aufgrund der Tatsache, daß bereits in den Vorlagen-Figuren etliche verschiedene Züge miteinander verschmolzen sind. Freilich kann man die Polystratie einer Figur als Autor kunstvoll mit noch mehr verschiedenen Strata anreichern, aber ob man wie Suerbaum (ebd.) wirklich behaupten kann, daß Vergils „Gestalten vielschichtiger als die Homers“ sind, das darf in dieser Generalität bezweifelt werden. 
terlassen ihre Spuren in mythischen Stoffen und damit auch in medialen Konkretisierungen wie z. B. Texten vor allem dann, wenn Taktiken erkennbar werden, die etwas mit Wertungen oder Hierarchisierungen zu tun haben (Kapitel 19.2). Altes, Neues oder auch schlicht Abweichendes wird entweder abgewertet oder in seiner Bedeutung heruntergestuft oder aufgewertet und hochgestuft. Dementsprechend besitzen mythische Figuren bestimmte Positionen in einem genealogischen oder hierarchischen Gefüge wie „Kind“ oder „König“ oder sich z. B. in konkreten Epitheta manifestierende Eigenschaften wie „übelgesonnen“ oder „,an Verstand überragend“ nicht an sich, sondern sie wurden ihnen auf dem Hintergrund von Deutungsmachtkonflikten zugeeignet und offenbaren ihre tiefere Bedeutung erst und vor allem dann, wenn man sie als relationale Aussagen begreift $^{4}$.

Eine solche Relationalität von Aussagen über mythische Figuren wird im Rahmen strukturalistischer Ansätze auf eine nicht unproblematische Weise übertragen auf bestimmte, feste Relationen zwischen mythischen Figuren selbst. So sollen bspw. nach Vernant die griechischen Gottheiten Hestia und Hermes implizit zwei gegensätzliche, sich komplementär ergänzende Aspekte der griechischen Vorstellung vom Raum verkörpern, und von daher als Figuren eng zusammengehören ${ }^{5}$ :

On peut dire que le couple Hermès-Hestia exprime, dans sa polarité, la tension qui se marque dans la représentation archaïque de l'espace : l'espace exige un centre, un point fixe, à valeur privilégiée, à partir duquel on puisse orienter et définir des directions, toutes différentes qualitativement ; mais l'espace se présente en même temps comme lieu du mouvement, ce qui implique une possibilité de transition et de passage de n'importe quel point à un autre.

Grundsätzlich schreibt Vernant an anderer Stelle ${ }^{6}$ :

Jeder Gott definiert sich durch das Beziehungsnetz, das ihn innerhalb eines bestimmten Pantheons mit anderen Gottheiten verbindet und von ihnen unterscheidet. Ein Element der mythischen Erzählung erhält Sinn nur durch seine Stelle im System, zu dem der Mythos gehört, dessen Bestandteil es ist.

4 S. dazu die beispielhaften Analysen im Kapitel 20.

5 Vernant, 1963, 15; zu weiteren Polaritäten der beiden Gottheiten ebd. 28, und zusammenfassend ebd. 47: „L'analyse des textes, qui mettaient l'accent sur les liens unissant le dieu et la déesse, a permis de dégager la relation de chacune de ces deux divinités avec des aspects définis et opposés de l'espace.“

6 Vernant, 1987, 134. 
Mythische Figuren und Stoffe sind tatsächlich miteinander vernetzt und aufeinander bezogen, aber in einem noch viel radikaleren Sinn und auf vielfältigere Weise als dies bei mancher strukturalistischen Konstruktion von Bedeutungszusammenhängen angenommen wird (s. dazu Kapitel 13.2). Wie die Synapsen im Gehirn nicht nur mit einem hauptsächlichen Partner, sondern vielfältig vernetzt sind, so auch mythische Figuren und Stoffe. Sowohl die Setzung von bestimmten, komplementär oder antithetisch zusammengehörigen Bezugspaaren ist problematisch und schwer zu beweisen, als auch schon die Grundannahme an sich, die darin besteht, daß die Figuren Hestia und Hermes jeweils in sich relativ einheitliche Konstrukte einzelner Aspekte kultureller Vorstellungen darstellen ${ }^{7}$.

Aufgrund der Polystratie mythischer Figuren folgt mit einer gewissen Notwendigkeit, daß mythische Figuren, und darunter besonders solche, die in mehreren verschiedenen und stärker verbreiteten Stoffen als Akteure auftreten, keine einheitlichen, sondern nicht selten auch inkonsistente Figuren sind ${ }^{8}$. Daß insbesondere eine wichtigere Gottheit verschiedenste, manchmal nicht einfach oder überhaupt nicht miteinander zu vereinbarende Genealogien, Herrschaftsbereiche, Zuständigkeiten oder „Charakterzüge“ hat, liegt eben an dem Umstand, daß es sich um eine polymorphe und im Einzelnen geschichtete Figur handelt, was den Entwurf eines einheitlichen, in sich konsistenten Bildes und damit eine einlinige Interpretation solcher Figuren a priori erschwert und zum Teil unmöglich macht ${ }^{9}$. Es gibt nicht und gab nie „den“ Hermes oder auch nicht „den“ Odysseus, sondern viele verschiedene „Hermesse“ und „Odysseuse“"10: Hermes, den Hirtengott, den Gott der Diebe und Händler, den Botengott, den Geleiter der Toten etc.;

7 Vgl. auch die Kritik an Vernant bei Powell, 2009, 42 f (besonders 43: „Objektivität und Bedeutung der Ergebnisse sind nur schwer zu bewerten ... praktisch überall anzuwenden ... ein zu ehrgeiziges Ziel“). Überzeugender, weil die Züge mythischer Figuren differenzierter nach verschiedenen Facetten analysierend, ist Vernants strukturalistische Interpretation der sich um Prometheus und Pandora rankenden mythischen Stoffe (Vernant, 1979, und 1987, 170-187); s. dazu auch Kapitel 17.1, S. 347.

8 Zur Inkonsistenz von mythischen Stoffen (bzw. Stoffvarianten) s. das Kapitel 16.

9 Das gilt für eine Figur vor allem, wenn man sie insgesamt betrachtet, etwas eingeschränkt hingegen für die Darstellung einer Figur in einer bestimmten medialen Konkretion einer einzelnen Stoffvariante, und für die Darstellung einer Figur in mehreren Stoffen, die aber von einem Autor in einem Werk vorgenommen wird. Auf einer solchen Konkretionsebene kann natürlich ein höheres Maß an innerer Konsistenz erreicht werden, auch wenn bezweifelt werden darf, daß man dabei gerade bei Homer so weit gehen kann wie Hirschberger, 2011, 278, wenn sie mit Bezug auf die Darstellung der Götter in Ilias und Odyssee schreibt: „Die Götter der homerischen Epen sind ... mythische Wesen mit jeweils ausgeprägter Persönlichkeit und distinktivem Charakter.“ 10 Im Plural von den mehreren „Atreusen, Thyesten, Agamemnonen und Aigisthossen“ (in der Tragödie) spricht im Übrigen bereits der antike Autor Ailianos (Ail. var. 2,11). 
Odysseus, den König von Ithaka, den kriegerischen Troiahelden, den piratenähnlichen Raubbeuter, den begabten Sänger, den raffinierten Trickster, den abenteuerlustigen Entdecker etc. ${ }^{11}$; und selbst, wenn man einen oder in Kombination mehrere dieser Aspekte herausgreift, so ist bspw. Hermes der Totengeleiter in der einen Stoffvariante nicht unbedingt identisch mit dem Totengeleiter Hermes, wie er in einer anderen Stoffvariante dargestellt wird. Es ist nicht "der“ Gott Hermes, der einmal die und überraschenderweise ein andermal eine ganz andere „Seite“ hat, sondern es sind zeitlich und räumlich verschiedene Hermes-Konzeptionen, die sich überlagern und z. T. durch die Inkonsistenzen im Einzelfall innerhalb einer bestimmten Stoffkonkretion wie auch erst recht in einem artifiziell zusammengeführten Gesamtbild nur allzu deutlich zu erkennen geben, daß es sich um eine polymorphe und je nach einzelner Darstellung auch geschichtete Figur handelt $^{12}$. Man kann fragen, wie Hermes in dieser oder jener Konkretion gestaltet ist, aber die Frage danach, wie losgelöst von einzelnen Konkretionen „der“ Hermes an und für sich beschaffen ist, führt notwendig zu einer künstlichen und von daher wenig befriedigenden, inkonsistenten Konstruktion, denn an und für sich gibt es „den“ Hermes nicht.

Im Bereich der bildlichen Darstellung mythischer Figuren läßt man sich durch die in manchen Fällen beobachtbare Konstanz beispielsweise von Götterattributen manchmal zu der Annahme unveränderlicher Hauptmerkmale einzelner Figuren und damit zum Postulat einer grundsätzlich gleichbleibenden Figurenzeichnung verleiten. So sieht etwa Junker in ikonographischer Hinsicht eine „feste Spezifik der Figuren“ als gegeben $\mathrm{an}^{13}$. Eine gewisse ikonographische Konstanz, die aufgrund der deutlich höheren Mehrdeutigkeit bei bildlichen Darstellungen von Figuren eher notwendig ist als bei textlichen, darf aber nicht dazu führen, eine solche Konstanz auch für die Einbindung dieser Figuren in verschiedene textliche Konkretionen mythischer Stoffvarianten vorauszusetzen, und auch bei bildlichen Darstellungen kommt es durchaus zu einem Wandel hinsichtlich der Attribute von Figuren, so daß eine „feste Spezifik der Figuren“ in vielen

$11 \mathrm{Zu}$ verschiedenen Odysseus-Figuren, mit denen Homer es zu tun hatte, die er durchblicken läßt, die er aber mit einer neuen Akzentsetzung auch überformt, s. resümierend auch Danek, $1998,508 \mathrm{f}$.

12 Vgl. grundsätzlich treffend bspw. Sourvinou-Inwood, 1987, 223; vgl. in Bezug auf die Göttergestalten in Homers Ilias bspw. Graf, 2002, 115: „Wie die Darstellung der Akteure auf der menschlichen Ebene ..., ist diejenige der Götter eingespannt in das Spannungsfeld von Tradition (im Sinne der vom Erzähler in seiner Umwelt vorgefundenen Erzählstoffe) und eigenem Gestalten.“ 13 S. Junker, 2005, 125; vgl. ebd. 125 f: ,Jede Gestalt des Mythos, die in eine bestimmte Handlung eingebunden ist, repräsentiert unabhängig von der wechselnden Situation, in der sie gerade erscheint, ein Set von unveränderlichen Eigenschaften.“ 
Fällen (wenn überhaupt) nur für einen zeitlich wie räumlich eng umgrenzten Bereich beobachtet werden kann.

Das Problem besteht nicht so sehr darin, daß man bislang zu wenig zu der Erkenntnis vorgedrungen wäre, daß mythische Figuren polymorphe und je nach einzelner Konkretion auch komplex geschichtete Gestalten sind (dies durchaus auch), sondern vor allem darin, daß es an Konsequenz bei der Umsetzung dieser Erkenntnis fehlt, daß trotz dieser Erkenntnis immer wieder der Versuchung nachgegeben wird, so etwas wie einen „Figurenkern“ oder eine „Urgestalt“ ausmachen zu wollen oder all die disparaten Elemente einer Figur zu einem bestimmten „Charakter“ zu synthetisieren - nicht selten begleitet von Verwunderung, wie schwierig sich eine solche Synthese im Einzelfall gestaltet. Exemplarisch ist etwa folgender Auszug aus van Dijks Deutung „der“ Göttin Innana-Ištar ${ }^{14}$ :

Es gibt im sumerisch-akkadischen Pantheon keine kompliziertere Gestalt als ... InannaIštar. Ihr wichtigster Charakterzug ist immer der eines Freudenmädchens ... Wie dša ra und ${ }^{d} l$ ú-làl ihre Söhne geworden sind, ist schwer zu erklären. ... Viel schwieriger ist es zu erklären, wie sie mit den gefürchteten Lamaštu-Dämoninnen gleichgestellt wurde. ... / Die kriegerische Inanna ist vor allem in der Akkad-Zeit ... sehr betont worden ... Man darf aber nicht verneinen, daß Inanna auch Vegetationsgöttin war, oder geworden ist. ... Inanna ist somit eine Persephone-Gestalt. Ein sicherer Synkretismus findet sich in der Götterliste An $=$ Anum,.. in der sie mit Antum, mit der Muttererde, Ninili usw. gleichgesetzt wird. ${ }^{15}$

Oder, um noch ein Beispiel aus der griechischen Mythologie anzuführen: Man liest zwar fast übereinstimmend zur Gestalt der Göttin Kirke: „Bien des éléments entrent dans cette personalité complexe“"16; Kirke sei ,a complex figure, patterned from many diverse elements“17 oder ,a composite figure, in whom elements of Near Eastern and Greek goddess worship are blended and unified“18. Doch fast im selben Atemzug geht man dann doch wieder dazu über, den Kern ihres „Charakters“ oder den „eigentlichen Ursprung“ dieser schillernden Gestalt näher zu bestimmen, wenn es z. B. bei Yarnall heißt: „The force of her character is not Indo-European but reminiscent of the potent, often terrifying female deities of

14 S. van Dijk, 1998, 9 f (mit der älteren Schreibung „Inanna“).

15 Deutlich differenzierter Wilcke, 1980, 79 f, nach dem die Frage, „ob es sich um nur eine Göttin handelt, die z.T. unter verschiedenen Namen oder mit besonderen Beinamen bedacht an vielen Orten verehrt wurde, oder ob es mehrere in ihrem Wesen oder ihrem Wirkungsbereich verschiedene Inanna/Ištar-Göttinnen gab“ unterschiedlich beantwortet werden muß ,je nach Zeit und Art der Quellen“ (Zitat ebd. 79).

16 Germain, 1954, 274.

17 Crane, 1988, 31.

18 Yarnall, 1994, 51. 
Asia Minor“, oder bei Germain: „Mais pour l'essentiel, la déesse aux chants aigus, aux amours redoutables, reste une Orientale“, verbunden mit der unbegründeten Behauptung, die mit der Gestalt der Kirke verbundene Idee, „que le contact féminin désarme et dégrade“, käme aus dem Orient ${ }^{19}$.

Es geht hier nicht darum, solche synthetisierenden Annäherungsversuche an die Komplexität mythischer Figuren in Bausch und Bogen zu verurteilen. Sie können im Einzelfall einen Erkenntnisgewinn bringen und als erste Orientierungshilfen dienen, um sich der Vielförmigkeit und Vielschichtigkeit dieser Figuren anzunähern. Problematisch wird es erst, wenn man meint, man könnte all die verschiedenen Puzzle-Teilchen zu einem in sich stimmigen Gesamtbild vereinigen, zu dem Bild einer Figur, wie sie „an und für sich“ oder „im eigentlichen Kern“ oder „von ihrem Ursprung her“ ist - und wenn man überdies aus uneinheitlichen Charakterzügen und Handlungsweisen mythischer Figuren falsche Folgerungen zieht.

Denn wenn in ein und derselben Quelle Inkonsistenzen hinsichtlich der inhaltlichen Gestaltung der Handlung oder in der Zeichnung der handelnden Figuren vorliegen, dann wird nur allzu häufig angenommen, daß verschiedene Autoren am Werk waren oder ein späterer Interpolator schwer Verständliches vermeintlich verbessert, in Wirklichkeit aber verunklart hat, oder daß einem Abschreiber Fehler bei der Textüberlieferung unterlaufen sind, oder daß ein Erzähler vermutet werden muß, der unfähig war, dem Stoff und seinen Figuren eine innere Konsistenz zu geben o. ä. ${ }^{20}$. Solche Inkonsistenzen können aber auch auf den Umstand zurückgeführt werden, daß die zur Verarbeitung anstehenden Stoffe und Figuren mit Ecken und Kanten versehen waren, die nicht restlos geglättet werden konnten oder sollten. Zu den Gründen und Motivationen, die für eine Beibehaltung von Inkonsistenzen verantwortlich gemacht werden können, und zu der rezeptionsästhetisch anderen Haltung, was Inkonsistenzen in mythischen Stoffvarianten anbelangt, sei hier auf die Ausführungen in den Kapiteln 16.1 und 16.2 zurückverwiesen. Hier kommt es darauf an, aus diesen Beobachtungen weitere Folgerungen für die Mytheninterpretation zu ziehen, gerade wenn es um eine Einordnung und Deutung mythischer Figuren und ihres Verhaltens geht.

19 Zitate jeweils auf denselben Seiten wie in den vorigen Anmerkungen angegeben.

20 S. dazu Kapitel 16.3. 


\subsection{Die unersättlich machtgierige Innana, oder: Die psychologische Falle der Mytheninterpretation}

Wenn es um psychologische Deutungen von Mythen geht, sind grundsätzlich zwei verschiedene Bereiche zu unterscheiden, auf welche sich solche Deutungsversuche beziehen können: zum einen die Ebene der Figuren, zum anderen die Ebene der Handlung. Im ersten Fall richtet die Interpretation ihr Augenmerk auf einzelne Figuren der Handlung und deren Verhalten, das unter psychologischen Gesichtspunkten analysiert wird. Eine solche Herangehensweise stellt etwa folgende Fragen: Warum handelt eine Figur so, wie sie handelt, was sind ihre inneren Motivationen, und auf welche geistig-seelischen Verfaßtheiten sind diese Motivationen zurückzuführen? Ein anderer Ansatz psychologischer Mythendeutung wird dort verfolgt, wo primär die Gesamthandlung eines mythischen Stoffes im Fokus steht, also die Figuren unter dem Aspekt ihrer wechselseitigen Beziehungen, die sich in bestimmten Verhaltensweisen oder Taten äußern.

$\mathrm{Zu}$ dem letztgenannten Ansatz zwei - eher willkürlich herausgegriffene Beispiele. So wird etwa von Yarnall die Verwandlung der Gefährten des Odysseus in Schweine durch die Göttin Kirke als ein gleichsam archetypisches Handeln der weiblichen Natur interpretiert, das die Unterlegenheit einzelner männlicher Wesen zum Ausdruck bringen soll ${ }^{21}$. Beim Stillen an der Mutterbrust hätten alle Männer diese Inferiorität schon erfahren. Odysseus aber sei gegen Kirkes Tücke vielleicht deshalb gefeit gewesen, so vermutet die Autorin, weil er in seiner Jugend bei einer Jagd am Parnaß-Gebirge durch die Hauer eines Ebers verletzt wurde und aufgrund einer solchen Stich-Verletzung durch ein Schwein sei er gewissermaßen gegen die Verwandlung in ein Schwein „symbolically“ geimpft gewesen ${ }^{22}$. Hinter der Handlung und Figuren-Konstellation des mythischen Stoffes wird also nach Yarnalls Ansicht die Aufarbeitung eines universal-anthropologischen Genderkonflikts erkennbar. Ein zweites, ähnlich gelagertes Beispiel ist etwa Miles' Deutung der Gestalt der Erdgöttin Gaia und der mit ihr verbundenen mythischen Stoffe als Widerspiegelung griechischer Männerängste vor feminin-matriarchaler Potenz ${ }^{23}$ : „Das Hin und Her von Fürsorge und Vernichtung in der Gestalt Gaias zeigt die Angst der griechischen Männer vor weiblicher und mütterlicher Macht.“

21 Yarnall, 1994, 20: „... the primordial feminine’s acting according to its own nature and making manifest the comparative inferiority of individual male being“.

22 Yarnall, 1994, 21: Odysseus sei gefeit, „perhaps, because he has already been pierced, symbolically inoculated, by the power of a pig“; die Autorin nimmt Bezug auf die Episode Hom. Od. 19,386-466. Zu einer eher funktional-kultischen Deutung bestimmter Handlungsweisen der Kirke-Gestalt s. ausführlich C. Zgoll, 2019a.

23 Geoffrey Miles in: Segal, 2016, 18. 
Auf die Frage nach der Fruchtbarkeit von Herangehensweisen, die hinter einem mythischen Stoff als Ganzem bestimmte psychologische Konstellationen oder Handlungsmuster erkennen, also im Jung’schen Sinn „Archetypisches“24, kann hier nicht näher eingegangen werden. Das, was mythischen Stoffen in manchen Fällen einen archetypischen Anstrich verleiht, ist der Umstand, daß den konkreten Stoffen manchmal Stoffschemata zugrundeliegen ${ }^{25}$, die typische oder zumindest als typisch angesehene Geschehnisse, Konstellationen oder Situationen beinhalten können ${ }^{26}$. Das heißt nicht automatisch, daß diese für mythische Stoffe und ihr Verständnis zentrale Bedeutungselemente darstellen müssen. $\mathrm{Ob}$ und inwieweit dies für die oben gegebenen (und andere) Beispiele zutrifft, soll hier nicht entschieden werden, sondern dem Urteil der Leserinnen und Leser überlassen bleiben ${ }^{27}$. Im Folgenden soll vor allem der andere Ansatz, nämlich

24 Vgl. Jung, 1952, 679 f: „Durch die Erforschung der Produkte des Unbewußten ergeben sich ... erkennbare Andeutungen archetypischer Strukturen, die mit den mythischen Motiven in eins fallen, und darunter gewisse Typen, die den Namen Dominanten verdienen: Es handelt sich um Archetypen wie Anima, Animus, alter Mann, Hexe, Schatten, Erdmutter etc. ...“ Vgl. ebd. 680 f: „Es ist ohne weiteres ersichtlich, daß die Kenntnis dieser Typen die Mythenbildung erheblich erleichtert und zugleich auf den Boden stellt, auf den sie gehört, nämlich auf die Grundlage der Psyche.“ Vgl. in Anlehnung an und Abgrenzung von Jung Kerényi, 1965, 243: „Was in solcher Unmittelbarkeit seinsgemäß-menschlich geschieht, dem eignet das griechische Eigenschaftswort archétypos, 'archetypisch', 'urbildhaft', 'prägend' - Mythen, Bilder, heilige Handlungen prägend und durch sie das Leben von Einzelnen und Gemeinschaften. Für sich existierende Archetypen und als Dimension für ihre Existenz das kollektive Unbewußte der Jungschen Psychologie werden da nicht gefordert.“ Vgl. Günther, 2013, 273, nach dessen Ansicht (die m. E. durch ein vorsichtigeres „manchmal“ etwas einzuschränken wäre) „Mythen nicht nur die erfahrbare Welt beschreiben oder erklären, sondern grundsätzliche, archetypische Merkmale und Paradigmen dieser Welt offenlegen. " Vgl. auch Drewermann, 2013, 15, für den die antiken Mythen auf lebenswichtige Fragen „mit ergreifenden Erzählungen“ antworten, „denen, in näherer Betrachtung, der Wert von Archetypen zukommt, deren Themen sich in einer Handvoll klassischer Konflikte konzentrieren.“

25 S. dazu Kapitel 7.3.

26 Vgl. bspw. Drewermann, 2013, 11: „,... unwichtig sind vergleichsweise die einzelnen Namen der Göttinnen und der Götter, Nymphen, Heroinen und Heroen, wichtig sind die Gestaltungen und Gestalten der Seele selbst, die in ihnen vor Augen treten.“ Mythenlektüre ist für Drewermann nur sinnvoll als Therapie für die Heilung des eigenen, sich nach Liebe sehnenden, von Leidenschaften aber auch bedrohten Ich, s. ebd. 14: „Das überzeitlich Gültige, das Typische des seelischen Erlebens, das die Mythen schildern, gilt es, zum Heilmittel all der Erkrankungen zu destillieren, zu synthetisieren, welche im Leben und Erleben Einzelner die Liebe immer wieder bis hin zu Wahn, Zerstörung und Verbrechen heraufzuführen fähig sind.“

27 Zur Bedeutung der von Freud angenommenen, für seine Interpretation von Träumen und Mythen gleichermaßen wichtigen universalen Symbole und überhaupt zum Themenkomplex „Mythos und Psychologie“ s. die ausführliche Darstellung bei Csapo, 2005, 80-131. Sehr luzide 
eine figurenpsychologische Deutung mythischer Stoffe, näher in den Blick genommen werden.

Wie bei mythischen Stoffen, so handelt es sich bei mythischen Figuren um komplexe, auf verschlungenen Pfaden und über lange Zeiten hinweg entstandene patchwork-Produkte, an denen viele mitgewirkt haben. Daraus folgt, daß bei dem Versuch, ein Gesamtbild von einer solchen Figur zu erstellen, ihr psychologisches Profil unscharf bleibt und nicht selten sogar mit leichten Widersprüchlichkeiten behaftet ist. Mit anderen Worten: In der Regel sind die Eigenschaften und v. a. die geschilderten psychischen Verfaßtheiten und Handlungsmotivationen einer mythischen Figur inkonsistent.

In diesem Punkt unterscheiden sich mythische Figuren fundamental von intentionalen Konstrukten einzelner Autoren. Ungeachtet aller Einflüsse von außen sind Handlungen und Helden etwa vieler neuzeitlicher oder moderner Romane in der Regel Erzeugnisse ihrer Autoren und weisen von daher normalerweise eine gewisse Einheitlichkeit und Konsistenz auf ${ }^{28}$. So wird von einem Roman etwa Dostojewski'scher Manier erwartet, daß die vom Autor geschaffenen Figuren bei aller Komplexität und sogar punktuellen Unberechenbarkeit in sich stimmig gezeichnet sind und handeln, und daß Veränderungen dieser Figuren sich organisch aus dem Handlungsverlauf ergeben oder doch zumindest im Prinzip psychologisch nachvollziehbar sind. Diese Erwartungen können von einem Romanautor um so mehr erfüllt werden, als er die auftretenden Figuren und den Ablauf der Handlung nach eigenem Ermessen gestalten kann ${ }^{29}$.

Ganz anders sieht die Angelegenheit aus, wenn ein Mythos erzählt wird. Hier treten mythische Figuren als Protagonisten auf, die in zahlreichen Liedern und sonstigen medialen Darstellungen längst existieren, und sie handeln als Protagonisten in Stoffen, die von jemandem, der diese Stoffe in welcher Variante und Form auch immer medial konkretisiert, nicht erfunden, sondern vorgefunden werden. Jeder, der bspw. die Stoffe, die sich um die Figur des Odysseus ranken,

und dabei ausgewogen zu den Chancen, v. a. aber auch zur Problematik bestimmter methodischer Vorannahmen einer psychoanalytischen Mythendeutung äußert sich Zinser, 1985. Zur Problematik von und Kritik an tiefenpsychologischen und psychoanalytischen Deutungsversuchen von Märchen, die sich analog auf entsprechende Deutungsversuche von mythischen Stoffen übertragen lassen, s. auch Neuhaus, 2005, 27-38. Zur Problematik der Annahme von „Archetypischem“ in mythischen Stoffen s. Kirk, 1980, 75 f.

28 S. dazu auch die Ausführungen in Kapitel 16.3.

$29 \mathrm{Zu}$ literarischen Werken als „intentionalen Gebilden“ vgl. Martínez/ Scheffel, 2012, 110, mit der Explikation, daß auffällige „Inkonsistenzen ... dem intentionalen Gebilde des literarischen Werkes nicht als funktionale Bestandteile zugerechnet werden“ können. Kommen solche Inkonsistenzen vor, sind sie „Zwar textgenetisch erklärbar, aber nicht textstrukturell interpretierbar“. 
zur Darstellung bringen will, steht vor der Herausforderung, die vielfältigen Stofftraditionen zu einer neuen Stoffvariante zu synthetisieren, die den eigenen und den Vorstellungen und Wünschen der Rezipienten entspricht, und aus den verschiedenen, bereits vorliegenden „Odysseusen“ einen, nämlich den im konkreten Fall gewünschten oder geforderten Odysseus zu machen. Statt der Freiheit, eine für einen selbst kreierten Stoff passende, eigene Figur zu erschaffen, besteht der Zwang, mit fremden und noch dazu komplex geschichteten Figuren und Stoffen zu arbeiten ${ }^{30}$. Nun kommt zu diesem Befund aber noch ein zweiter, entscheidender Punkt hinzu:

$\rightarrow \quad$ Figuren und Stoffe sind im Falle von Mythen nicht nur polymorph und je nach Einzelfall polystrat, sondern sie sind auch nicht speziell aufeinander abgestimmt.

Auch darin unterscheiden sie sich wesentlich von literarischen Figuren und Stoffen etwa moderner Romane. Insofern fiktive Personen in modernen Romanen intentionale Konstrukte einzelner Autoren darstellen, handelt es sich automatisch um Erzeugnisse, die sich in aller Regel in eine angenommene Hauptintention des Werkes einfügen und somit auf den Inhalt der Handlung speziell zugeschnitten $\operatorname{sind}^{31}$. Dasselbe kann bei mythischen Figuren nicht vorausgesetzt werden. In mythischen Stoffen vorkommende Figuren führen ein Eigenleben, das nicht mit einem oder auch mehreren Stoffen untrennbar verbunden ist. Im Extremfall wird eine mythische Figur in einen ihr völlig fremden Stoff hineingesetzt, wie sich oben am Beispiel des assyrischen Stadt- und Staatsgottes Aššur gezeigt hat, der in den Aššur-Versionen des Enūma eliš nun plötzlich anstelle von Marduk die

30 Man kann natürlich, auch wenn dies wenig überzeugend erscheint, aus der Not eine Tugend machen und gewissermaßen durch einen Kunstgriff die Widersprüchlichkeiten und Gegensätze einer gewachsenen, vielschichtigen Figur kurzerhand zum Programm erklären, durch welches „das griechische Imaginäre, das bei der Konstituierung der Heros-Figur am Werk ist“ absichtsvoll eine Einheit der Gegensätze konstruiert, wie dies bspw. Loraux, 1985 (Zitat ebd. 170), mit der Figur des Herakles unternimmt, und die daher vorschlägt (vgl. ebd., 169), „... den heroischen Charakter durch seine Widersprüche hindurch als eine Einheit zu definieren, dessen Identität gerade durch diese Widersprüche konstituiert ist.“ Das führt Loraux u. a. zu der „Behauptung, daß wir in Herakles eine der griechischen Figuren vor uns haben, die für die Weiblichkeit im Manne stehen“ (ebd. 178).

31 Zum „hypothetischen Intentionalismus“ als Interpretationsansatz, der auf „eine von historischen Plausibilitätskriterien eingeschränkte Vielzahl von Bedeutungszuschreibungen“ abzielt bzw. auf ein „'Feld' von Bedeutungen, die von dem Verfasser tatsächlich hätten gemeint sein können“, s. Spoerhase, 2007, 123-144; Zitate ebd. 125. 
Meeresgöttin Tiāmtu bekämpft und besiegt oder die Welt erschafft ${ }^{32}$. Und der Gott Hermes spielt in verschiedenen mythischen Stoffen eine wichtige Rolle, ohne daß man sagen könnte, daß Hermes speziell für diese Stoffe oder umgekehrt all diese verschiedenen Stoffe speziell für die Gestalt des Hermes gemacht worden seien.

Aus diesen Beobachtungen ergibt sich eine Folgerung, die für die Interpretation und Bewertung von mythischen Figuren und ihren Handlungen grundlegend wichtig ist. Die Motivation für bestimmte Handlungsweisen einer einzelnen Figur in mythischen Stoffen bzw. konkret vorliegenden Stoffvarianten richtet sich nicht hauptsächlich nach einer bestimmten, für die jeweilige Figur typischen Psyche, weil eine polymorphe und polystrate mythische Figur eine solche für sie typische Psyche gar nicht besitzt, sondern sie richtet sich primär nach den Erfordernissen, die sich aus der jeweiligen Thematik oder Problematik des mythischen Stoffes ergeben, mit dem die mythische Figur im Einzelfall verknüpft ist ${ }^{33}$. Mit anderen Worten:

$\rightarrow \quad$ Mythische Stoffe dienen nicht der Profilierung einer unverwechselbaren Psyche einer in ihnen auftretenden Figur, aus der sich deren Handlungsmotivationen ableiten ließen, sondern die Thematik oder Problematik eines Stoffes diktiert maßgeblich die Handlungsweise und Darstellung der Figuren $^{34}$.

32 S. dazu Kapitel 20.10.

33 Mit Blick auf Märchenstoffe wurde die Nachrangigkeit der Figurenpsychologie bereits von Propp und Lüthi herausgearbeitet. Nach Propp, 1975, 75, verleihen die verschiedenen Beweggründe und Absichten der handelnden Figuren zwar „dem Märchen bisweilen ein ganz besonderes Kolorit, dennoch gehören sie zu den allervariabelsten und unbeständigsten Märchenelementen. ... Die meisten Aktionen der handelnden Personen des Mittelteils eines Märchens sind naturgemäß durch den Gang der Handlung bedingt ...“Vgl. ebd. 81: „Hier stoßen wir wieder auf die Tatsache, daß Wille und Absichten der Gestalten kein entscheidendes Kriterium für ihre funktionsmäßige Einordnung sind. Wichtig sind nicht ihre wahren Absichten oder Gefühle, sondern ihre Handlungen an sich unter dem Aspekt ihrer Bedeutung für ... den Gang der Handlung.“ Vgl. auch Lüthi, 2004, 28: „Personen und Dinge des Märchens sind im allgemeinen nicht individuell gezeichnet.“ Ebd. 30 der Hinweis, „daß die Handlung des Märchens nicht von innen gelenkt wird, sondern von außen.“ Lüthi fährt fort: „Das heißt, es wird alles möglichst auf die gleiche Fläche projiziert, auf die der Handlung; alles dahinter Stehende bleibt so gut wie unbeleuchtet. Innenleben (Gefühl, Stimmung, Anstrengung) und Umwelt der Figuren ... spielen eine ... geringe Rolle ...“

34 Vgl. mit Bezug auf Homer Burgess, 2015, 44: „... it is important to realize that these [sc. die Hauptfiguren in den homerischen Epen] are not real people with a full psychology. Their portrayal is based on their actions in traditional myth, supplemented by rare descriptions and hints by the poet ...“ Wenn Burgess im Folgenden dann doch kurze Charakterporträts erstellt, dann 
Wiederum erweist sich hier die Unterscheidung zwischen dem Stoff und der Konkretion einer Stoffvariante wie z. B. in Form eines bestimmten Textes als wichtig. Es geht hier um die Stoffebene, nicht um die Textebene einer einzelnen Stoffvariante. Es kann durchaus sein, daß ein einzelner Autor einen bestimmten Stoff in der Konkretion einer bestimmten Stoffvariante dazu benutzt, eine Art Psychogramm einer mythischen Figur zu entwerfen, das mehr oder weniger konsistent erscheint. So kann die Konkretion einer Stoffvariante im Einzelfall durchaus für eine psychologisierende Charakterzeichnung einer mythischen Figur funktionalisiert werden, wie bspw. Lukian in seinen Götterdialogen zahlreiche mythische Stoffe und Figuren gerade dadurch wunderbar ironisiert, daß er in seinen konkreten Stoffgestaltungen die Handlungen der Figuren auf menschlich-allzumenschliche Verhaltensweisen herunterbricht und bestimmte Taten rein psychologisch motiviert sein läßt, so daß diese psychologisch motivierten Verhaltensweisen in diesem begrenzten Rahmen zu einer durchaus konsistenten Charakterzeichnung einer Figur führen können. Diese Ebene der Stoffkonkretionen und ihrer im Einzelfall sehr unterschiedlichen Funktionalisierungen soll keineswegs ausgeblendet werden ${ }^{35}$. Aber hier geht es nicht um die Ebene einzelner Konkretionen von Stoffvarianten in ihrer jeweiligen, medial unterschiedlichen Endgestalt, sondern um die Ebene des mythischen Stoffes als offene Menge der möglichen Stoffvarianten insgesamt, und auf dieser Ebene spielt das Psychologische eine deutlich untergeordnete Rolle.

Wenn mythische Stoffe nicht der psychologischen Charakterisierung und Individualisierung der Figuren, sondern vielmehr umgekehrt die Figuren den Erfordernissen dienen, die sich aus der Thematik oder Problematik eines Stoffes ergeben, dann bedeutet das wiederum:

geht es ihm nicht um „den“ Charakter von bspw. Achilleus an sich, sondern um die Charakterzeichnung, die Achilleus bei Homer erhält. Vgl. auch Lotman, 1972, 357-368, der den Versuch einer grundsätzlichen Abkehr von der Rede des „Charakters“ literarischer Figuren und der Rückführung von Figuren-Eigenschaften auf ein kultur- und auch jeweils textspezifisches Paradigma unternimmt, s. ebd. 356: „Der Charakter einer Figur ist die Summe aller im Text gegebenen binären Oppositionen zu anderen Figuren ... Der Charakter ist also ein Paradigma.“ Und ebd. 367: „Somit hängt die Rückführung verschiedener Erwähnungen irgendeiner Person im Text auf eine einheitliche paradigmahafte Gestalt immer von dem jeweiligen Kulturcode ab, und zwar sowohl für den Autor wie für sein Auditorium.“ Vgl. dagegen bspw. den Versuch einer psychologisierenden Charakterzeichnung der Göttin Innana/ Ištar in jüngster Zeit bei Schneider, 2015, 61: „Ishtar's interest is in sexuality, not marriage, either for herself or others. What drives her is adrenaline and excitement ...“

35 Zur Wichtigkeit, nach einer Hylem- und Stratifikationsanalyse wieder das Gesamt einer konkret vorliegenden Stoffvariante in den Blick zu nehmen, s. die Ausführungen in Kapitel 21.1. 
$\rightarrow \quad$ Mythische Stoffe sind nicht primär an der Aufarbeitung der psychologischen Ursachen und Motive einer Figurenhandlung interessiert, sondern an den Handlungen selbst bzw. an den durch die Handlungen anvisierten Zielen und Ergebnissen.

Wenn es der im Stoff behandelten Problematik dienlich ist, können mythische Figuren deshalb im Einzelfall auch sonderbare oder im Vergleich mit etlichen anderen Konkretionen ihrer Gestalt eher untypische Haltungen, Eigenschaften oder Handlungen zugeschrieben bekommen.

Trotz der vorgestellten Überlegungen zur Nachrangigkeit psychologischer Faktoren ist es nun aber unabweisbar so, daß in mythischen Stoffvarianten Figurenhandlungen immer wieder psychologisch motiviert erscheinen, wobei unter „psychologisch“ hier verstanden wird, daß solche Handlungen auf bestimmte seelische Regungen, emotionale Antriebe oder charakterbedingte Züge zurückgeführt werden. In dieser Hinsicht nähern sich vor allem textliche Konkretionen mythischer Stoffvarianten der Gattung „Roman“ durchaus an.

Darin besteht aber gerade eine besondere Gefahr, denn diese Gemeinsamkeit täuscht darüber hinweg, daß die Rolle, die das Psychologische jeweils spielt, gänzlich verschieden bewertet werden muß. So sind etwa in den Romanen Dostojewskis die psychologischen Hintergründe, aus denen heraus die Figuren agieren, ein Element mit fundamentaler Bedeutung für den Zusammenhang und die Erklärung der Handlung. Mit ihnen steht und fällt der plot, und hat man die psychologischen Hintergründe erklärt, hat man damit zugleich etwas Wesentliches, wenn nicht das Wesentliche der betreffenden Werke erfaßt und interpretiert. In mythischen Stoffvarianten (und ihren medialen, v. a. textlichen Konkretionen) hingegen kommt Psychologisches zum einen allein deswegen vor, weil in kaum einem Erzählstoff auf eine psychologische Motivierung in der Handlungsführung völlig verzichtet werden kann, und zum anderen, weil in entsprechend gestalteten Stoffvarianten für die Rezipienten ein nicht unbeträchtliches Genußpotential liegt - aber nicht, weil das Psychologische eine auch nur annähernd vergleichbare, tragende Funktion für Verständnis und Interpretation des Rezipierten besitzt.

Problematisch wird die Angelegenheit dann, wenn man genau dies voraussetzt, nämlich daß die Figurenpsychologie in mythischen Stoffvarianten eine ebenso wichtige Rolle für die Interpretation dieser Stoffe spielen würde, wie dies bei vielen Romanen oder etwa auch Kriminalgeschichten der Fall ist, wenn man also meint, man hätte einen mythischen Stoff oder einen einzelnen Zug daraus verstanden bzw. erklärt, indem man auf bestimmte psychologische Handlungs- 
motivationen aufmerksam macht. Ein solches Vorgehen ist gleichermaßen verlockend wie verfehlt und soll hier als die „psychologische Falle“ der Mytheninterpretation bezeichnet werden.

Wenn man beispielsweise erklärt, Hera habe Io deswegen mit Hilfe einer Bremse von Griechenland bis nach Ägypten fast zu Tode gehetzt, weil sie auf ihren Ehemann Zeus, der mit Io eine Affäre hatte, eifersüchtig war, so hat man nur die oberflächliche Handlungsmotivation beschrieben, also ein Hylem, in dem von der Eifersucht der Hera die Rede ist, zwar methodisch richtig extrahiert und als einzelnen Stoffbaustein isoliert, aber so gut wie nichts von dem erklärt, worum es in diesem mythischen Stoff eigentlich geht. Ohne eine platzgreifende Interpretation des Io-Mythos auszubreiten sei hier wenigstens in äußerster Verknappung angedeutet, daß man näher an die Bedeutung wenigstens eines Aspektes des Stoffes gelangt, wenn man auf das Ergebnis der „Liebesaffäre“ zwischen Zeus und Io, auf Epaphos, und auf die schon bei Herodot belegbare Parallelisierung der Gestalt der Io mit der ägyptischen Göttin Isis und des Epaphos mit dem ägyptischen Apis hinweist ${ }^{36}$, so daß die Flucht der Io ausgerechnet nach Ägypten kein Zufall ist und Eifersucht als handlungsauslösendes Moment sich somit in diesem speziellen Fall als stoffliche Einkleidung eher religionshistorischer (oder kultischer $^{37}$ ) Zusammenhänge bzw. Phänomene entpuppt und daher nicht primär darauf abzielt, die Götterkönigin Hera als unangenehm zu charakterisieren und ihre „böse“ Handlungsweise zu „erklären“38. Psychologisierende Deutungsversuche von Figurenhandlungen stehen in der Gefahr, sich als Interpretationen einer tieferen Bedeutungsebene von mythischen Stoffen zu verstehen, obgleich sie nur die Oberfläche der Stoffgestaltung betreffen.

Figurenpsychologie in Mythen ist in der Regel nicht Selbstzweck, sondern Mittel zum Zweck. Da angesichts des Umstandes, daß mythische Figuren und Stoffe nicht aufeinander abgestimmt sind, in der Erarbeitung figurenpsychologischer Mechanismen a priori nicht das Kernanliegen mythischer Stoffe erblickt werden kann, ist davon auszugehen, daß Darstellungen von psychologischen Verhaltensweisen oder typischen Konstellationen in mythischen Stoffen nicht in erster Linie deshalb vorkommen, weil es um diese psychologischen Gegebenheiten selbst geht, sondern weil mit ihrer Hilfe transzendierende Auseinandersetzun-

36 Hdt. 2,41,2 und 3,28,2.

37 So Burkert, 1997, 186 f, mit Verweis auf Riten des Herakultes in Argos.

38 Eine eingehendere Deutung des Stoffes würde hier zu weit abführen; vgl. Ansätze dazu etwa bei Burkert, 1997, 185-187. Weiterführende Literatur bei Reinhardt, 2011, 243, Anm. 913, und Reinhardt, 2016, 40. 
gen mit als bedeutsam eingestuften Erfahrungsgegenständen auf besonders ansprechende, spannende oder drastische Weise stofflich verdichtet werden können. Es ist somit neben der annähernden Unmöglichkeit, Handlungen in einem Erzählstoff völlig ohne psychologische Motivationen zu gestalten, primär der Unterhaltungswert (und daneben auch noch der mnemotechnische Vorteil einer leichteren Merkbarkeit), der dazu führt, daß Figurenpsychologie bei der Ausgestaltung mythischer Stoffe zum Einsatz kommt.

Daß eine figurenpsychologische Perspektive wiederum auch bei einer Interpretation von Mythen oft zu sehr in den Fokus rückt, dafür ist ein anderer Faktor verantwortlich zu machen, und zwar der Faktor einer prinzipiell leichten oder zumindest für leicht gehaltenen Übertragbarkeit geschilderter psychologischer Mechanismen auf die eigene Erfahrungswelt der jeweiligen Interpreten. Vom Verhalten mythischer Protagonisten lassen sich schnell Brücken zu eigenen Erlebnissen, Emotionen, Verhaltensweisen schlagen, und genau darin liegt sowohl die Gefahr wie auch die Ursache dafür, daß man bei psychologisierenden Interpretationsversuchen, den unterschiedlichen psychologischen Richtungen und auch den verschiedenen eigenen psychischen Erfahrungen und Einstellungen entsprechend, zu recht disparaten Ergebnissen gelangt.

Nicht nur disparat, sondern zum Teil regelrecht konträr sind bspw. die meistenteils psychologisierenden Deutungsversuche, die in Bezug auf den Mythos von Enlil und Ninlil zu finden sind, so daß sich die bekannte und vernichtende Kritik, diese Deutungsversuche könnten mehr über die Deuter als über das Gedeutete sagen, schwer ganz von der Hand weisen läßt ${ }^{39}$. Die in diesem Mythos beschriebenen Sexualakte und ihre Folgen bzw. die beteiligten Figuren werden etwa von Black et al. folgendermaßen interpretiert ${ }^{40}$ : „The psychology of this is complex. Enlil seems to want to escape from Ninlil. Ninlil seems willing to have sex with anyone. Or is it all a complex sexual game?" Bottéro und Kramer sehen hingegen in Enlil und Ninlil, „même s'ils n’ont pas été 'régulièrement' mariés, ... un couple authentique“, und das, was der Mythos zeige, sei „précisément cette union stable et conjugale qui a fait d'Enlil et de Ninlil de vrais parents“"41. An anderer Stelle wird vermutet, daß ,die Defloration einer freien Jungfrau ohne Wissen und Einwilligung der Eltern und Brautjungfern“ die Götter deshalb sehr erzürnt und zu einer Bestrafung Enlils geführt habe, weil dieses Vergehen ,außer-

39 S. zu diesem Mythos ausführlicher Kapitel 21.2, mit Anm. 13.

40 Black/ Cunningham/ Robson/ Zólyomi, 2004, 102.

41 Bottéro/ Kramer, 1993, 114. 
halb der Stadt begangen wurde, wo das Mädchen sich weder verteidigen noch um Hilfe rufen konnte“42.

Um zu verdeutlichen, wie verlockend die psychologische Falle der Mytheninterpretation ist und wie sehr sie trotz solcher disparaten Ergebnisse die Forschungen zu Mythen bestimmt, sollen noch einige wenige weitere Beispiele angeführt werden. So wird etwa der Vorgang der Verzauberung der Gefährten des Odysseus durch Kirke und deren spätere Befreiung durch Odysseus einmal dadurch ,erklärt“, daß die auf ihrer einsamen Insel wohnende Göttin „spröde“ gewesen sei und sich durch Verzauberung von Eindringlingen „gegen jede männliche Berührung“ gewehrt habe, und daß erst Odysseus ,ihr sexuell imponieren und sie zum Beischlaf bekehren" konnte ${ }^{43}$. An anderer Stelle findet sich folgende allegorisierend-psychologisierende Deutung der Gestalt der Kirke und der Verzauberung der Odysseusgefährten in Schweine ${ }^{44}$ : „Die Mutation der Gefährten ins Borstenvieh wird zur poetisch-sinnlichen, zu unmittelbarer Kenntlichkeit entstellten Erscheinung ihres animalischen Wesens, das die ihrerseits in eine kultivierte Nobelhetäre verwandelte Dämonin scheinbar unwiderstehlich unter der Schicht einer zweckgerichteten Rationalität hervorzog ...“

Wie wichtig die Dimension der Figurenpsychologie ist, um die Rezipienten $\mathrm{zu}$ fesseln und zu faszinieren, das hat unter anderen Ovid erkannt und sich diese Dimension immer wieder, vor allem aber natürlich in den Metamorphosen, für die farbige und faszinierende Ausgestaltung mythischer Stoffe zunutze gemacht, so daß bei Ovid nicht mehr so sehr die Handlungsverläufe an sich, nicht Götter und ihre Taten und ihr aktives Eingreifen in die Welt der Menschen im Zentrum stehen, sondern die Reaktionen der Menschen und die menschliche Psyche, deren Beschreibung meisterhaft differenziert wird, und die auch dadurch zum heimlichen Zentrum der Stoffgestaltungen avanciert, daß nicht mehr so sehr Psychologisches im Dienst der Handlung, als vielmehr die Handlung im Dienst der Psychologie steht und auch die Götter und ihr Handeln ganz aus der Perspektive menschlicher psychischer Verfaßtheiten und Motivationen geschildert werden. Um mythische Stoffe in ihren Konkretionen zu genießen, ist die Dimension der

42 Steible, 2015, 23. Nach weiteren Deutungen von Enlil und Ninlil, die von einer reinen Figurenpsychologie weggehen und schon in eine ,archetypische“ Deutung hineinspielen, zeigt die Erzählung bspw. „die ambivalente emotionale Gemengelage von Vergewaltigungsopfern gegenüber dem Täter“ (vgl. Cooper, 1980), oder geht es vielmehr darum, anhand der Ninlil „die Entwicklung vom Mädchen zur Mutter vieler Kinder“ nachzuzeichnen (vgl. Leick, 1991, 48); zusammenfassende Zitate der Forschungen von Cooper und Leick nach A. Zgoll, 2013, 87, mit entsprechenden Literaturhinweisen.

43 Mannsperger, B. und D., 2006, 141.

44 Rohdich, 2003, 2. 
Figurenpsychologie also durchaus wichtig und wertvoll. Um Mythen aber zu verstehen und zu erklären, dafür trägt die Durchleuchtung psychologischer Handlungsmotivationen wenig bei und kann sogar irreführend sein, indem sie das Augenmerk vom Eigentlichen auf Oberflächliches ablenkt und zudem dazu verleitet, Verhaltensweisen mythischer Protagonisten zu sehr auf dem Hintergrund eigener Erfahrungen zu interpretieren. Bereits bei Müller findet sich die treffende Feststellung, daß psychologisierende Handlungsmotivationen mythischen Stoffen weitgehend fremd sind und vorrangig in dichterischen Bearbeitungen auftauchen, und daß gerade die dann jeweils unterschiedlich ausfallenden Deutungen von psychologischen Handlungsmotivationen ein weiteres Indiz für ihre nur lose Verbindung mit den Stoffen liefern ${ }^{45}$.

Daß es sich bei der figurenpsychologischen Dimension in mythischen Stoffen um etwas eher Nachrangiges handelt, geht nicht zuletzt auch daraus hervor, daß in etlichen Fällen, ganz im Gegensatz zu vielen Romanen oder Kriminalgeschichten, eine psychologische Motivation der Handlungsweise mythischer Figuren völlig fehlen kann, obwohl die Handlung so merkwürdig oder herausragend ist, daß man eine solche Erklärung eigentlich erwarten könnte. Die Erwartungshaltung, daß Handlungen von Figuren psychologisch oder zumindest überhaupt irgendwie motiviert werden, ist aber bei vielen und v. a. modernen Rezipienten so selbstverständlich und so stark ausgeprägt, daß man nicht selten dem Phänomen begegnet, daß bei Interpretationen von Mythen psychologische Handlungsmotivationen dort, wo man ihr Fehlen zu sehr vermißt, nachträglich in den Stoff hineingetragen werden.

Bei Homer etwa steht, daß die Göttin Kirke die Gefährten des Odysseus in Schweine verwandelt hat; es ist aber nirgends explizit etwas darüber ausgesagt, warum Kirke so handelt. Prompt kommt es zu dem oben zitierten Versuch, diese Handlung nachträglich bspw. durch die Erfindung einer sexuellen Sprödigkeit der Göttin männlichen Eindringlingen gegenüber zu erklären. Einen anderen prominenten Fall stellt der Mythos vom Gang der Göttin Innana/ Ištar ins Totenreich dar. Eine der höchsten Göttinnen im mesopotamischen Pantheon macht sich auf, um in das Totenreich zu gehen. Aber warum? Die textlichen Konkretionen dieses in sumerischer und akkadischer Sprache überlieferten mythischen Stoffes sagen darüber nichts aus. Und auch hier wird diese Lücke wieder aufgefüllt, indem man der Göttin psychologisierend bspw. Stolz und Herrschsucht unterstellt ${ }^{46}$ : „No explanation is offered why Inana should take it into her head to

45 Vgl. Müller, 1825, $208 \mathrm{f}$.

46 So bei Black/ Cunningham/ Robson/ Zólyomi, 2004, 65. Vgl. ähnlich Waetzold, 2015, 375 und 377: „Dieser Mythos berichtet vom vergeblichen Versuch der Göttin Inanna, die Macht über die 
seek domination over the Underworld. The fierce, ambitious aspect of her character and her insatiable desire for power are reasons enough.“

Mit solchen von außen hineinprojizierten Psychologisierungen ist nicht nur nichts gewonnen, sondern in ihnen liegen vielmehr Abwege und sogar Gefahren. So werden mit Hilfe psychologisierender Deutungsversuche zum Teil auch Inkonsistenzen übertüncht. Im Mythos von Innanas Gang zur Unterwelt, in einer Variante textlich konkretisiert im sumerischen Lied von Innanas Gang ins Totenreich $^{47}$, gerät die Göttin in der Unterwelt in wahrhaft „tödliche“ Gefahr und muß sich am Ende durch die Stellung einer Ersatzperson freikaufen. Da der von ihr geliebte Dumuzi nicht um die verloren geglaubte Göttin trauert, sondern im Festgewand auf seinem Thron sitzt, gerät Innana in Zorn und bestimmt ihn dazu, an ihrer Stelle in das Totenreich zu gehen. Wenig später aber ist unvermittelt davon die Rede, daß Innana um Dumuzi trauert und nach seinem Verbleib fragt. Der Umschwung in Innanas Verhalten findet keine Erklärung im Text und damit auch nicht auf Stoffebene, aber durchaus eine problematische, weil figurenpsychologische Erklärung von seiten der Forschung ${ }^{48}$ : „Aus tiefer Enttäuschung und Wut, daß Dumuzi nicht wie die anderen Gottheiten um sie trauerte, hatte sie ihn den (Unterwelt-)Schergen überlassen. Jetzt spürt sie die Folgen, sehnt sich nach ihm und beginnt, ihn zu suchen.“

Es braucht an dieser Stelle nicht mehr betont werden, daß man mit einer solchen Glättung von Inkonsistenzen wichtige Hinweise auf verschiedene Stoffstrata verdeckt. Wenn im Text von Innana explizit ausgesagt wird, daß sie nicht wisse, wohin ihr Geliebter verschwunden ist und eine Fliege ausschickt, um nach ihm zu suchen ${ }^{49}$, obwohl vorher davon die Rede war, daß sie selbst ihn den Totenreichs-Schergen übergeben hat ${ }^{50}$, dann ist das ein deutlicher Hinweis auf alternative und in diesem Fall sogar tatsächlich nachweisbare Stofftraditionen ${ }^{51}$, nach denen Innana nicht selbst für Dumuzis Auslieferung verantwortlich war -

Unterwelt zu gewinnen. ... / Von ihrer Überheblichkeit und den hochfahrenden Plänen bleibt nichts übrig.“

47 S. zum Text und seinen Bearbeitungen Anm. 27 in Kapitel 12.4.

48 Waetzold, 2015, 377.

49 Innanas Gang ins Totenreich Z. 390-399.

50 Innanas Gang ins Totenreich Z. 348-367.

51 Verschiedene, in unterschiedlichen Texten greifbare Stoffe benennen verschiedene Ursachen dafür, daß Dumuzi ins Totenreich gehen muß, und lassen von daher ein komplexes Bild erkennen; so wird Dumuzi etwa nach dem Text Innana und Bilulu durch Bilulu und ihren Sohn ermordet, nach dem Text Dumuzis Tod gelangt er durch einen Sturz in eine Erdspalte in die Unterwelt, und Klagelieder wiederum machen Dämonen dafür verantwortlich. 
nicht auf einen plötzlichen psychologischen Stimmungsumschwung oder gar trauerbedingten, partiellen Gedächtnisverlust der Göttin.

\subsection{Sex mit einer Schlafenden: Die naturalistische Falle der Mytheninterpretation}

Handlungen von Protagonisten in Mythen müssen nicht nur psychologischen, sondern auch üblichen physiologischen Gegebenheiten bzw. tatsächlichen (oder geglaubten) Naturgesetzen nicht entsprechen:

$\rightarrow \quad$ Wie am Psychologischen, so sind Mythen auch am Physiologischen und an Naturgesetzlichkeiten nicht primär interessiert, sondern physiologische oder naturhafte Vorgänge werden vorrangig als Mittel zum Zweck der Erreichung bestimmter Ziele eingesetzt.

Diese Vorrangigkeit der Ziele äußert sich u. a. darin, daß für die Erreichung derselben physiologische oder naturhafte Vorgänge durchaus so gestaltet sein können, daß sie üblichen und beobachtbaren Gegebenheiten und Gesetzlichkeiten nicht entsprechen oder ihnen sogar zuwiderlaufen ${ }^{52}$. Eine Interpretation, die sich darauf konzentriert, diese Merkwürdigkeiten zu erklären, anstatt $\mathrm{zu}$ fragen, $z u$ welchem Zweck diese Merkwürdigkeiten funktional eingesetzt werden, gleicht dem Versuch, das Wesen einer Windmühle durch eine ausführliche Beschreibung der auffälligen Windmühlenflügel zu erfassen, ohne daß in den Blick kommt, wozu diese Windmühlenflügel sich eigentlich drehen. Nicht auf die Windmühlenflügel kommt es an, sondern auf das Mehl, das am Ende herauskommt, und dafür ist es vergleichsweise egal, mit Hilfe welcher merkwürdiger Konstruktionen dieses Mehl nun gemahlen wurde. Ein solche mißleitende Fokussierung auf physiologische oder naturhafte Merkwürdigkeiten führt in die „naturalistische Falle“ der Mytheninterpretation.

Als Beispiel soll der sexuelle Verkehr mit einer schlafenden Frau in mythischen Stoffen angeführt werden, wenn also etwa im sumerischen Text Innana und Šukaletuda der „Gärtner“ Šukaletuda mit der schlafenden Innana ${ }^{53}$ oder der

52 Vergleichbare Beobachtungen lassen sich auch bei Märchenstoffen machen und werden in der Märchenforschung u. a. mit Begriffen wie „Wunder“ oder „Phantastik“ in Zusammenhang gebracht. Vgl. Lüthi, 2004, 99, der meint, die „Verbindung von Wirklichkeit und Phantastik“ sei „für das Märchen überhaupt charakteristisch“; vgl. auch Neuhaus, 2005, 373, der in Bezug auf Märchen „das zentrale Merkmal des Wunderbaren“ unterstreicht.

53 S. zum Text und seinen Bearbeitungen Anm. 28 in Kapitel 12.4. 
Kriegsgott Mars mit der schlafenden Ilia sexuell verkehrt ${ }^{54}$. Wichtig ist nicht die Frage, wie das ohne ein Erwachen von seiten der Frau - ohne daß dabei Drogen oder Alkohol im Spiel wären - physiologisch konkret ausgesehen und funktioniert haben soll, sondern der Umstand, daß ein Beischlaf stattgefunden hat, und für die Interpretation dieser Stoffe entscheidend ist vor allem der Blick auf das Ergebnis dieses Vorganges, im Fall von Mars und Ilia nämlich die Geburt der Gründer von Rom, Romulus und Remus, und die dahinterstehende Aussage, daß die Gründer-Zwillinge sich direkt aus einem göttlichem Ursprung ableiten, der im Fall der kriegerischen Römer auch nicht zufällig im Kriegsgott Mars erblickt wird. Was den Text von Innana und Šukaletuda anbelangt, so ist dort weit weniger die Vorstellung von einem sexuellen Verkehr mit einer Frau merkwürdig, die eben eingeschlafen ist und offenbar von dem Sexualakt nichts mitbekommt ${ }^{55}$, als vielmehr der Umstand, daß in dieser textlichen Konkretion des dahinterliegenden mythischen Stoffes nicht genannt wird, zu welchem Zweck dieser sexuelle Verkehr initiiert wurde. In der Suche nach einer Antwort auf diese Frage dürfte ein wichtiger Schlüssel für die Interpretation des Stoffes liegen, und der wird sicherlich nicht darin bestehen, daß man den Gärtner Šukaletuda als einen eben besonders lüsternen Mann zu charakterisieren versucht. Wenn Šukaletuda am Ende für den Beischlaf mit der Göttin bestraft wird, so dürfte der Grund am ehesten darin zu suchen sein, daß er durch den Verkehr mit der Göttin dieser etwas Wichtiges geraubt bzw. für sich etwas bekommen hat, von der Fruchtbarkeitsgöttin Innana vielleicht Fruchtbarkeit für seinen Garten, so wie Prometheus unter Strafe das für die Menschen so wichtige Feuer von den Göttern stiehlt - und nicht etwa darin, daß Innana morgens nach dem Erwachen frustriert darüber zürnt, daß Šukaletuda nicht mehr da ist und sie sich deshalb von ihm zurückgewiesen fühlt, oder daß sie ihn deswegen straft, weil er ihren Ansprüchen nicht gerecht zu werden vermochte ${ }^{56}$.

Um nur noch ein weiteres Beispiel anzuführen: Für den Mythos von Enlil und Ninlil ist es entscheidend darzustellen, $d a \beta$ vier wichtige Gottheiten aus der Verbindung gerade dieser beiden Hochgottheiten des sumerischen Pantheons hervorgegangen sind, der Mondgott, die mit der Unterwelt assoziierten Gottheiten

54 Vgl. dazu die Darstellung bei Ov. fast. 3,9-22.

55 So Mittermayer, 2013, 37: „Es ist schwer zu glauben, dass sie einen Moment später bereits tief schlafen soll, ohne etwas von dem Sexualakt wahrzunehmen.“

56 Vgl. diesen zwar verständlichen, aber eher an Romanen als an der Funktionsweise mythischer Stoffe orientierten Versuch bei Mittermayer, 2013, 37, Anm. 28, die nach dem Beischlaf von Innana vorgenommene Bestrafung des Šukaletuda psychologisierend zu erklären: „Fühlte sich Innana von Šukaletuda zurückgewiesen, weil er am Morgen nicht mehr anwesend war, oder war er ihren Ansprüchen nicht gerecht geworden?“ 
Nergal und Ninazu, und der für Bewässerungskanäle wichtige Gott Enbilulu, und daß durch diese stoffliche Darstellung ein hierarchisches Beziehungsgeflecht etabliert wird, das in Übereinstimmung mit theologischen Vorstellungen Zusammengehörigkeiten und Ränge verschiedener Gottheiten klärt ${ }^{57}$. Das physiologische „Problem“, daß Ninlil dafür in kürzestem Abstand viermal hintereinander schwanger wird und gebiert, ist demgegenüber ebenso ohne Belang wie die Frage nach psychologischen Motivationen oder Dispositionen der handelnden Figuren $^{58}$.

57 S. zu diesem Mythos Kapitel 21.2, mit Anm. 13.

58 Vgl. dazu die leicht verwunderte Feststellung bei Black/ Cunningham/ Robson/ Zólyomi, 2004,102: „There seems to be no concern over the obvious problem of serial pregnancy and multiple births, which here seem to override natural laws.“ Zu psychologisierenden Deutungen dieses Mythos s. das vorige Kapitel. 